\title{
Dodecagonal order in a two-dimensional Lennard-Jones system
}

\author{
P. W. Leung \\ Laboratory of Atomic and Solid State Physics, Cornell University, Ithaca, New York 14853 \\ Christopher L. Henley \\ Laboratory of Atomic and Solid State Physics, Cornell University, Ithaca, New York 14853 \\ and Department of Physics, Boston University, Boston, Massachusetts 02215* \\ G. V. Chester \\ Laboratory of Atomic and Solid State Physics, Cornell University, Ithaca, New York 14853 \\ (Received 9 May 1988)
}

\begin{abstract}
We investigate a two-dimensional Lennard-Jones mixture with the interaction parameters chosen so as to favor configurations where the large atoms form squares and equilateral triangles. Many such configurations are possible which by our choice of interactions are nearly degenerate in energy. It is hypothesized that a thermal equilibrium state with 12 -fold orientational order exists. Several Monte Carlo simulations were performed to cool the system to a temperature approaching zero. The ordering process was studied by following the evolution of the configurations with temperature. The onset of ordering seemed to be very diffuse in space rather than nucleated at a point. The resulting configurations consist of squares and triangles, except for a few dislocations, and thus have perfect orientational order. We also characterized the deviation from ideal quasiperiodicity in terms of the "phason strain"; this was analyzed both by fitting a linear relation between the physical space coordinates of the atoms and the corresponding "perpendicular space" coordinates, and also by calculating the diffraction peaks. The latter are shifted and broadened, relative to an ideal 12 fold diffraction pattern, as in real quasicrystals.
\end{abstract}

\section{INTRODUCTION}

Though traditional solid-state physics and crystallography are founded on the assumption that the ground state of a set of atoms is periodic, demonstrating this is a very deep, nontrivial problem. This problem has become sharper with the discovery of various metallic alloys that form nonperiodic structure evidencing fairly sharp diffraction patterns with noncrystallographic symmetries-octagonal, ${ }^{1}$ decagonal, ${ }^{2}$ dodecagonal, ${ }^{3,4}$ or icosahedral. 5,6

The sharpness of their diffraction patterns suggests that long-range translational order exists in these materials. Early efforts have been focused on how to describe this long-range order in a nonperiodic structure. The deterministic Penrose tiling ${ }^{7}$ which has perfect longrange translational and orientational order turns out to be a natural candidate. But this ideal structure has matching rules that are hard to enforce by local packing of atoms. ${ }^{8}$ This leads to the hypothesis ${ }^{9}$ that quasicrystal formation is due to entropy effects: either a difference in thermodynamic (static) entropy, in the case of an equilibrium quasicrystal such as Al-Cu-Li (Refs. 6 and 10) or kinetic entropy, in the sense that there are more paths in configuration spacing leading to a metastable quasicrystal state such as Al-Mn (Refs. 11-13) thus favoring its formation in rapid quenching. The entropic hypothesis implies that the quasicrystal state is intrinsically disordered, in agreement with experiment. ${ }^{14}$

Our present work was motivated by this hypothesis. We sought a model physical system which would have a quasicrystal phase in thermodynamic equilibrium, stabilized by its higher entropy. Since simulations are faster and easier to visualize in two dimensions, for the present we consider two-dimensional models only.

Stephens and Goldman ${ }^{15}$ have proposed the "icosahedral glass" model which in two dimensions is a packing of rigid pentagons with neighboring pentagons sharing a common edge. Elser ${ }^{11}$ has studied the packing of two-dimensional decagons subjected to some special packing rules. In both of these models, the basic units of the packings (pentagons and decagons, respectively) represent rigid clusters of tightly bound atoms, which then connect together in a discrete, angular dependent way. Decagonal ordering is enforced in these systems by their packing rules.

These clusters are two-dimensional analogs of icosahedral clusters used in models of the quasicrystal atomic structure. ${ }^{16-18}$ However, there is, so far, no evidence that the atoms are more tightly bound within these clusters than between them. Thus, the basic assumption of the cluster packing models is very artificial. A model based on atoms is more desirable because it requires neither the rigid clusters nor the artifical packing rules to enforce the ordering. Furthermore, to study nucleation and growth of quasicrystals will require modeling the ordering process on an atomic scale. Hence, it is important to develop the techniques for attaining equilibrium in such systems, even though we are studying toy models without any detailed correspondence to the realistic structures.

Since a one-component system of atoms orders into a 
simple lattice, ${ }^{19}$ we are led to study two-dimensional two-component systems which have many nearly degenerate ground states, in each of which an orientational order is preserved. Lançon et al. ${ }^{20}$ and Widom et al. ${ }^{21}$ have studied such a two-component mixture which shows tenfold symmetry. Although they used slightly different forms of interaction potentials, their ground-state structures are essentially identical. In this paper, we adopt another such model which develops 12-fold symmetry.

This paper is organized as follows. In Sec. II A, we discuss the square and triangle tiling and its relation to dodecagonal quasicrystals. In Sec. II B we describe our atomic model which we used to simulate the square and triangle tiling, and Sec. II C explains our choices for boundary conditions, Monte Carlo dynamics, and interaction strengths. The remainder of the paper analyses the results: Section III A discusses the freezing mechanism that we observed, and in Sec. III B we define and discuss the possible topological defects that can develop in our model. Section III C and Sec. III D characterize the deviation of the final configuration from quasiperiodicity in terms of the "phason strain" and the diffraction pattern.

\section{MODEL}

\section{A. Square and triangle tiling}

Frank and Kasper ${ }^{22}$ have proposed hypothetical structures in which some layers of atoms form a packing of squares and triangles in a periodic way. These FrankKasper phases were later found in some Ni-based compounds. ${ }^{23}$ Two dimensional 12-fold quasicrystals have been observed in $\mathrm{Ni}-\mathrm{Cr}$ films ${ }^{3}$ which coexist with these Frank-Kasper phases. It was suggested ${ }^{3,24}$ that their structures consist of a packing of squares, triangles, rhombi, and hexagons. Recently, a new-type of twodimensional quasicrystals was found in $\mathrm{V}-\mathrm{Ni}$ alloys ${ }^{25}$ whose high-resolution electron-microscopy (HREM) image shows a packing consisting almost entirely of squares and triangles. Hence, square and triangle tiling is closely related to dodecagonal quasicrystals.

A two-dimensional 12-fold quasiperiodic lattice can be constructed by the projection method. ${ }^{26,27}$ In this case, the higher dimensional structure is a direct product of two triangular lattices in two dimensions. This fourdimensional periodic lattice is projected onto a twodimensional plane which is incommensurate. The resulting quasilattice consists of squares, triangles, and $30^{\circ}$ rhombi. To get a quasiperiodic lattice without the rhombi, one can use the method of inflation. ${ }^{28}$ By using a rational approximant ${ }^{16}$ to $\sqrt{3}$, we have constructed a periodic lattice of squares and triangles as shown in Fig. 1 (a).

The statistical mechanics of square and triangle tilings was studied by Kawamura ${ }^{29}$ in the context of amorphous systems. Since the bonds in such a lattice are of equal length and in discrete directions, orientational order is enforced. The properties of a square and triangle tiling depends on the number ratio of squares to triangles $N_{\text {sq }} / N_{\text {tr }}$. It can be shown that for a random tiling, $N_{\mathrm{sq}} / N_{\mathrm{tr}}=(\sqrt{3} / 4)$ (see Appendix A). In this paper, we aim to simulate a random square and triangle tiling. It is interesting to note that Kawamura found the maximum entropy at $T=0$ for $N_{\mathrm{sq}} / N_{\mathrm{tr}}$ to be approximately 0.45 , which is closed to $\sqrt{3} / 4$.

\section{B. Atoms and interactions}

Our two-dimensional system consists of two kinds of atoms - large $(L)$ and small $(S)$, interacting through a truncated Lennard-Jones potential

$V_{i j}(r)=\left\{\begin{array}{l}4 \epsilon_{i j}\left[\left[\frac{\sigma_{i j}}{r}\right]^{1 / 2}-\left[\frac{\sigma_{i j}}{r}\right]^{6}\right], \text { if } r \leq 3 \sigma_{i j} \\ 0, \text { otherwise , }\end{array}\right.$

where $i j=L L$ or $L S$. The length scales are chosen in such a way that they encourage a square and triangle tiling

$$
\begin{aligned}
& \sigma_{L L}=1, \\
& \sigma_{L S}=\frac{1}{\sqrt{2}} .
\end{aligned}
$$

Clearly, the ground state of a system containing only $L$ atoms is a triangular lattice. If we ignore the tail effect of the interactions, the ground state of an equal mixture of $L$ and $S$ atoms is a square lattice of $L$ 's with $S$ atoms at the centers, which means both the $L L$ and $L S$ nearest neighbors can simultaneously be at the minimum of their respective interactions. For intermediate concentrations of $S$ atoms, any packing of space by squares and equilateral triangles of equal edges with $L$ atoms on the vertices and $S$ atoms in the squares has practically the same energy as the ground state. The role of the small atoms is to stabilize the squares only. They are ignored in later analysis. We assign a short-range repulsive force between them

$$
\begin{aligned}
& V_{S S}(r)= \begin{cases}\epsilon_{S S}\left(\frac{\sigma_{S S}}{r}\right)^{1 / 2}, & \text { if } r \leq 2.5 \sigma_{S S} \\
0, & \text { otherwise },\end{cases} \\
& \sigma_{S S}=1 .
\end{aligned}
$$

The purpose of this repulsive force is to prevent more than one small atom from occupying a given interstitial space between large atoms. Equations (1), (2), and (3) define the model except for the numerical values of $\epsilon_{L L}$, $\epsilon_{L S}$, and $\epsilon_{S S}$. These are chosen so as to make the system mimic a random tiling of squares and triangles, as we are going to discuss in the next section. Figure 1(b) shows the decoration of the square and triangle tiling in Fig. 1(a). It consists of 209 large particles and 97 small particles.

In the same way we used the squares and triangles, Lançon et al. ${ }^{20}$ and Widom et al. ${ }^{21}$ used a decoration of the Penrose tiling to fix their interaction parameters of their system. Our model atoms have somewhat more realistic interaction parameters than compared with those of this tenfold symmetric model. Our ratio $\sigma_{L S} / \sigma_{L L}=1 / \sqrt{2}$ is closer to unity (as with real atoms) 
than the ratio $\sigma_{S S} / \sigma_{L L} \simeq 1.9$ in their model, and so our large particles have coordination numbers 6 to 8 , as compared to 5 to 10 in the other model. It is interesting to note that our repulsive interaction that forbids $S S$ neighbors is actually reminiscent of the interactions between $\mathrm{Mn}$ atoms in $\mathrm{Al}$ systems, where the $\mathrm{Mn}-\mathrm{Mn}$ neighbors are strongly disfavored in the known atomic structures. Finally, we remark that Widom et al. ${ }^{30}$ independently suggested a similar model.

\section{Monte Carlo details}

We put 280 large atoms and 120 small atoms into a box with rigid walls: any attempted move of a particle across
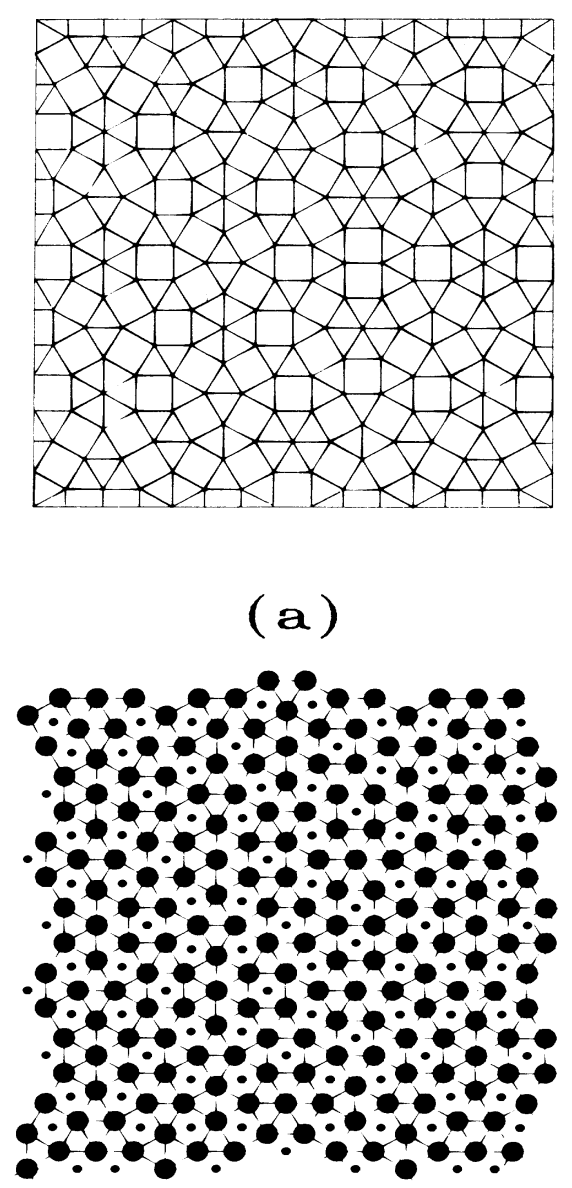

( b )

FIG. 1. (a) A periodic square and triangle tiling constructed by the method of inflation. It is a square cell of sides $(2+\sqrt{3})^{2} \sigma$ where $\sigma$ is the sides of the squares and triangles. (b) A unit cell of the tiling decorated by large and small particles. Large particles (large black circles) are placed at the vertices and small particles (small black circles) are placed at the center of the squares. It has 209 large particles and 97 small particles. Note that periodic boundary condition of the cell is taken into account. the boundary of the box was rejected. The area of the box is $800 \sigma_{L L}^{2}$. In the frozen phase, the solid occupied less than $40 \%$ of the total area. Thus, the solid, when formed, was subjected to free boundary conditions. This kind of boundary condition has the advantage that it is easier for defects to anneal out during the freezing process, ${ }^{21}$ although the result is more strongly affected by the finite-size effects.

In an infinite square and triangle tiling with 12-fold orientational symmetry, it can be shown that the number ratio of squares to triangles is precisely $(\sqrt{3} / 4)$ (see Ref. 9 and Appendix A). Provided that all the squares are occupied by $S$ atoms, it follows that the ideal bulk ratio of large to small atoms $N_{L} / N_{S}$, is $1+(2 / \sqrt{3})=2.155$. The use of free boundary condition introduces some complications. If $\epsilon_{L S} / \epsilon_{L L}$ is too small, the system tends to freeze to a three phase coexistent state consisting of a pure triangular lattice of large atoms, a solid phase of squares and triangles, and a fluid phase of small atoms around the solid. If $\epsilon_{L S} / \epsilon_{L L}$ is too large, all small atoms are surrounded by large atoms, leaving no small atoms at the boundary after they cohere to form a solid. For intermediate values, $\epsilon_{L S} / \epsilon_{L L}$ controls the amount of small atoms in the surface layer. Therefore, the bulk relative concentration differs from the total relative concentration $N_{L} / N_{S}$ by a correction proportional to the surface to bulk ratio. This is a kind of finite-size effect introduced by the free boundary condition, and is fairly large in our small system. We choose $\epsilon_{L S} / \epsilon_{L L}$ such that the system freezes to a state of squares and triangles with their number ratio close to the ideal value of $\sqrt{3} / 4$. It is also found that large $\epsilon_{S S}$ can favor dodecagons of six squares and twelve triangles, but at the price of disfavoring neighboring squares, since the $S$ atoms in neighboring squares are separated by $\sigma_{L L}$ only. But for the sake of randomness, we try not to discourage adjacent squares. ${ }^{31}$ The interaction parameters we have chosen are

$$
\begin{aligned}
& \epsilon_{L L}=1, \\
& \epsilon_{L S}=0.75, \\
& \epsilon_{S S}=1 .
\end{aligned}
$$

We start from an equilibrium configuration at

$$
T^{*}=k_{B} T / \epsilon_{L L}=0.5,
$$

which is in the fluid phase, and reduce the temperature by a factor of 0.95 every 10000 passes until it reaches a temperature of 0.338 . The temperature is then reduced by a factor of 0.95 every 20000 passes. At each temperature, the maximum trial displacements of each particle are adjusted to give an average acceptance rate of about 0.5 . The maximum trial displacements range from about $0.3 \sigma_{L L}$ for the small particles and about $0.2 \sigma_{L L}$ for the large particles at $T^{*}=0.338$ to about $0.09 \sigma_{L L}$ for the small particles and about $0.08 \sigma_{L L}$ for the large particles at $T^{*}=0.105$. In order to sample a larger phase space, each particle is given an attempted long jump, i.e., a move to any point inside the box with equal probability, every ten conventional Monte Carlo moves. When no rearrangement of the atoms is observed, the system is 
quenched to a temperature approaching absolute zero. This last step is to minimize the distortion of the squares and triangles due to thermal motions. During the process, the states of the system are characterized by calculating the internal energy $E_{\text {pot }}$ and the 12-fold orientational order parameter $\psi_{12}$ defined as

$$
\psi_{12}=\frac{1}{N}\left\langle\left|\sum_{i=1}^{N} \frac{1}{n_{i}} \sum_{\mathrm{NN}} e^{12 i \theta_{i j}}\right|\right\rangle,
$$

where $\Sigma_{\mathrm{NN}}$ means sum over the nearest neighbors, $n_{i}$ is the number of nearest neighbors of particle $i$, and $\theta_{i j}$ is the angle between the position vectors of particles $i$ and $j$. The structure factor,

$$
S(\mathbf{q})=\frac{1}{N^{2}}\left|\sum_{i=1}^{N} e^{i \mathbf{q} \cdot \mathbf{x}_{i}}\right|^{2},
$$

is also calculated to help monitoring the development of ordering.

There is a major difference between the behavior of our system and that of Widom et al. ${ }^{21}$ We presume that the important rearrangements are those which lead from one square and triangle tiling to another one; the same thing would be true with a different tiling for their model. But in their case, there exist tilings which differ by very local rearrangements (exchange of one of their large for two of their small atoms). It is believed that all the tilinglike configurations can be generated by a sequence of these moves which they have encouraged by an appropriate special Monte Carlo rule that exchange two neighboring small atoms with one large atom. On the other hand, the smallest subset of a square and triangle tiling which can be rearranged, leaving the rest fixed, is a chain of width two cells (see Fig. 2). The activation energy is finite but large-closed chains exist, but the smallest of these is a dodecagon of 12 triangles and 6 squares requiring the rearrangement of $6 L$ and $6 S$ atoms. Hence, we must anticipate much glassier behavior.

It is interesting to note that a similar difficulty exists in some three-dimensional icosahedral structures. Arbitrary tilings of Ammann rhombohedra can be reshuffled

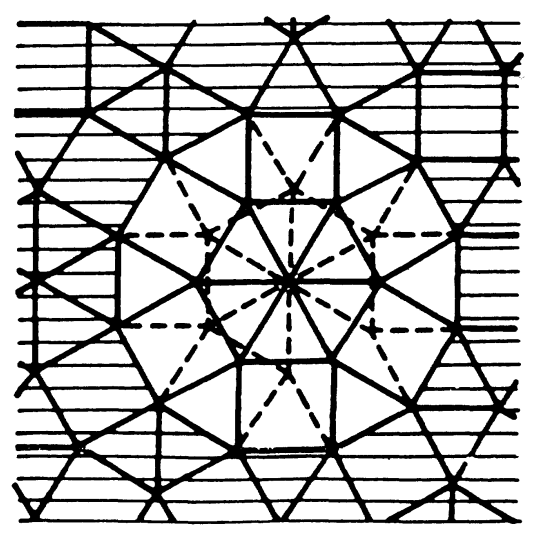

FIG. 2. The smallest closed chain that can be rearranged in a square and triangle tiling. It involves the rearrangement of six large particles and six small particles. (From Ref. 33). by moving one vertex at a time in the interior of a rhombic dodecahedron." 82 However, the tiling of "canonical cells, ${ }^{33}$ which have edges along three-fold and two-fold symmetry directions as inspired by the real structure, appears to suffer the same sort of difficulty as the square and triangle tiling.

\section{RESULTS}

\section{A. Freezing process}

As the temperature is lowered, the atoms began to aggregate. At the same time, internal rearrangement can occur in those places where the local network of the large atoms is neither square nor triangle. This is made possible by the use of the free boundary condition which reduces the relaxation time and the use of the nonlocal long jumps which causes a relatively large change in the local environment. The melting curves are plotted in Fig. 3. A rather continuous transition is observed. Figure 4(a) shows a snapshot of the system at $T^{*}=0.24$, where ordering is starting to appear. Eventually, the atoms approach their optimal separations. Figure 4(b) shows the final configuration at a temperature approaching absolute zero.

Here we should remark on the ordering mechanism that we have observed. The system develops local order in bond lengths at relatively high temperatures where the square and triangle structure is not stable. If it is rapidly quenched, locally the atoms will tend to collapse into squares and triangles but with an extremely high density of defects. These tend to be topological defects of the network, rather than vacancies or interstitials which are quickly healed by the long-range jumps. As noted earlier, the defects are very slow to anneal out in this model. Therefore, it is an interesting question how the system can find a state as well ordered as Fig. 4(b). In particular, why do not differently oriented domains form, and bond together, freezing in lines of defects? There are two conceivable explanations.

(1) If nucleation is infrequent and growth is rapid, then the quasicrystal phase would start from one seed and quickly grow, consuming all the disordered phase: the resulting structure has a good order because it all grows (a)

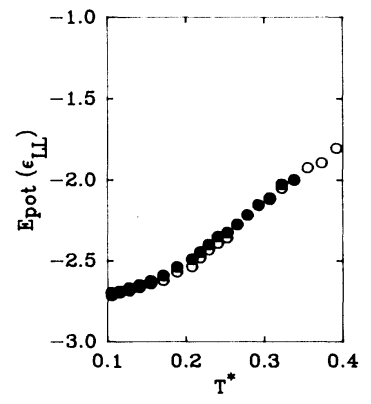

(b)

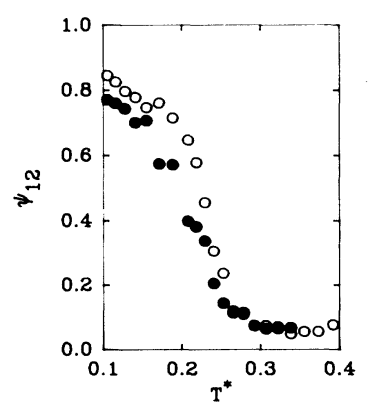

FIG. 3. (a) Internal energy $E_{\text {pot }}$ and (b) orientational order parameter $\psi_{12}$ at different temperatures during the freezing process. represents the freezing process and $\bigcirc$ represents the melting process. 

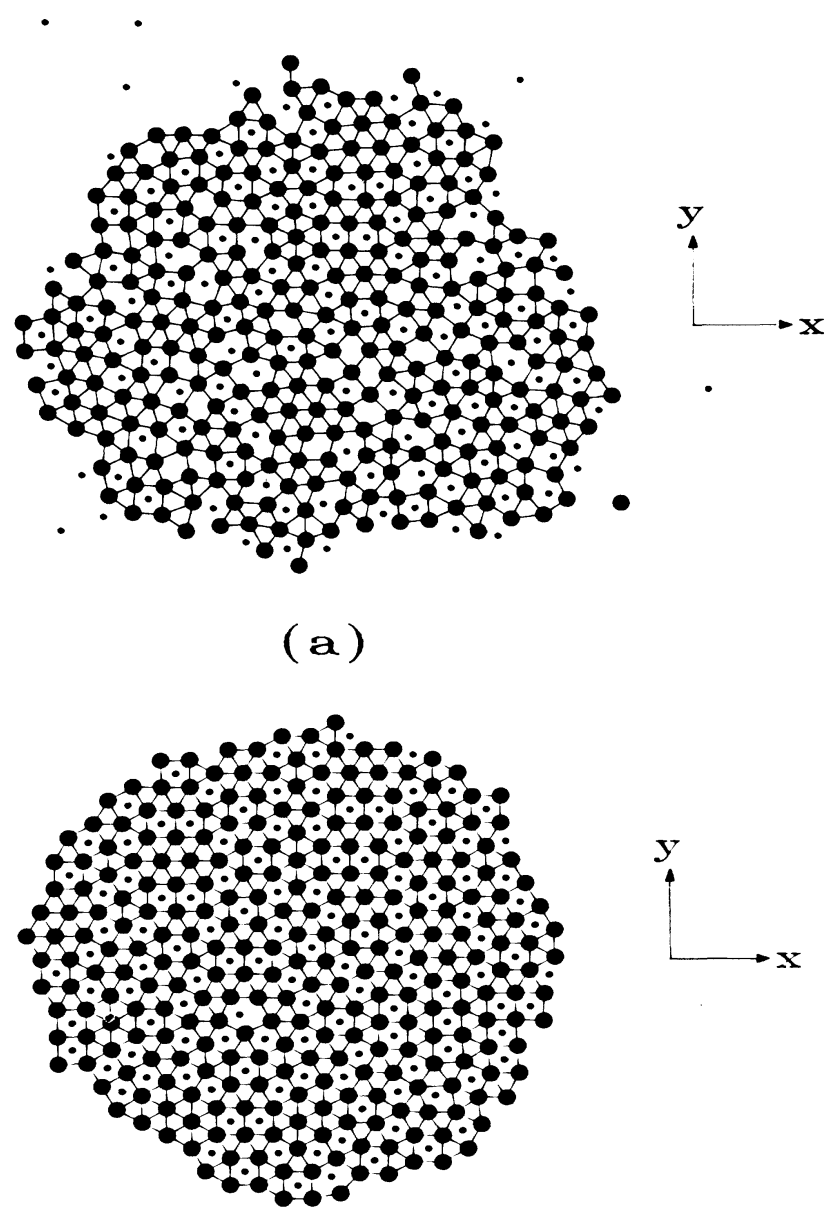

(b)

FIG. 4. Configurations at (a) $T^{*}=0.24$ when ordering is starting to appear, and (b) $T^{*}$ approaching zero. Neighboring large particles are joined by straight lines.

from a single point.

(2) On the other hand, the order may develop in a diffuse, nonlocalized way so that distant parts of the sample develop correlations when the network is still fluid enough to anneal out defects.

Our observations of "snapshots" of the configurations at the temperatures where order has its onset suggest the second, nonlocalized mechanism for the localization. This is a different kind of freezing mechanism from the one observed in a two-dimensional one-component Lennard-Jones system. In such a system, different regions develop local order with different orientations, which eventually align and merge together. ${ }^{34}$ But due to the finite-size effects in our small system, we cannot say what will happen in larger systems.

\section{B. Topological defects}

Various kinds of defects can appear in such a system. Vacancies and interstitials are common in a rapidly quenched system, but they are not important in our analysis, since in our case they can be eliminated through the slow cooling rate and the nonlocal long-range jumps. Phason strains are defects in the sense that they characterize departure from an ideal tiling; ${ }^{35}$ they will be discussed in the next section. Here we concentrate on topological defects. As in ordinary crystals, there are two kinds of topological defects in quasicrystals, disclinations, and dislocations. ${ }^{36,12}$

A disclination is defined quantitatively in the following way.

(1) Join all the neighboring large particles. Here neighbors are defined by a cutoff radius of about $1.3 \sigma_{L L}$. This will define a network of polygons. Some are triangles, some are quadrilaterals, and are designated "squares"; some will be pentagons or more exotic shapes and these shapes are subdivided, somewhat arbitrarily, into "triangles" and "squares." Thus, we define a network where each polygon is topologically a triangle or square; if it is possible to replace all of them by equilateral triangles and regular squares, we say there are no topological defects.

(2) For each lattice point, count the number of squares $n_{\mathrm{sq}}$ and triangles $n_{\mathrm{tr}}$ which share it as a common vertex. In the ideal case the nominal angles around it should add up to $2 \pi$. If not, it is said to be a disclination of strength $n$ where

$$
(2 \pi)(n / 12)=(2 \pi)\left(n_{\mathrm{sq}} / 4+n_{\mathrm{tr}} / 6-1\right) .
$$

Figure 5(a) shows a pair of disclinations of equal and opposite strength. Note that this definition of disclination does not require the squares and triangles to be perfect, since thermal motion will always distort the angles. It is clear that these are topological defects exactly like disclinations in ordinary two-dimensional crystals; the angle in (7) is the change in orientation when we take a dodecagonal frame along a closed loop of bonds by parallel transport, and so it is independent of which loop we take. The topological nature of the disclination also justifies the arbitrary procedure for defining it: if we made another choice the disclinations would be in slightly different places but the total disclination number within a loop would be the same. [See Figs. 5(a) and 5(b)].

A dislocation is defined as a neighboring pair of disclinations with equal and opposite strengths. We can also construct the Burgers vector for a dislocation (or group of dislocations) in a way analogous to the usual lattice circuit. ${ }^{37}$ We take the sum of the ideal vectors corresponding to the bond vectors around the loop enclosing the dislocations, and this gives the familiar Burgers vector

$$
\mathbf{B}=\sum_{i} b_{i} \widehat{\mathbf{e}}_{i}
$$

Because the basis vectors are incommensurate due to the noncrystallographic symmetry, it is common to have a nonzero Burgers vector shorter than a near-neighbor distance. Figure 5(c) shows the lattice circuit and Burgers vector for the dislocation in Fig. 5(a).

The dislocation is a topological defect and this justifies the arbitrariness in grouping disclinations into pairs. Note also that the disclination does not depend on using an ideal tiling as a reference structure, but it is well 
defined even in a random tiling structure. If a structure is free of dislocations, this does not imply that it is an ideal quasiperiodic tiling; it only means that we can replace all of the topological triangles and squares in the bond network, which were distorted by thermal vibrations, by rigid equilateral triangles and squares without any conflict.

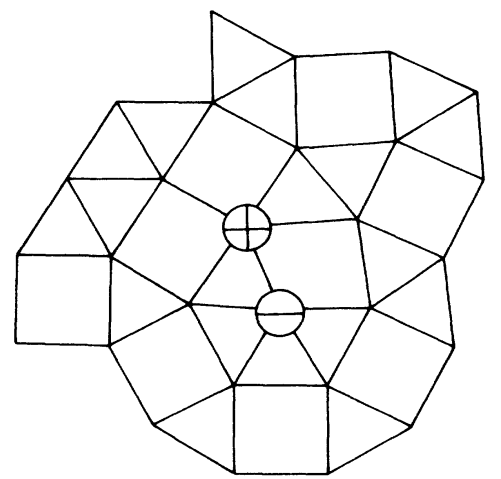

(a)

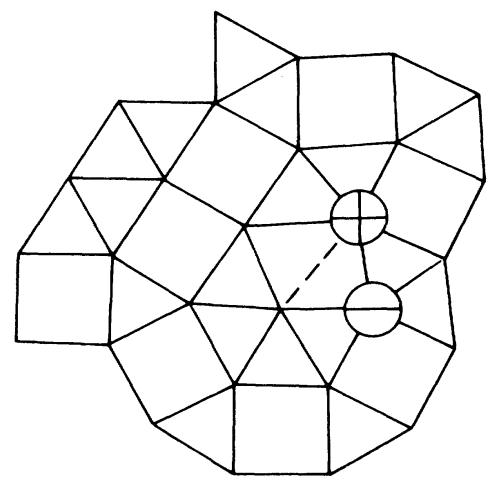

(b)

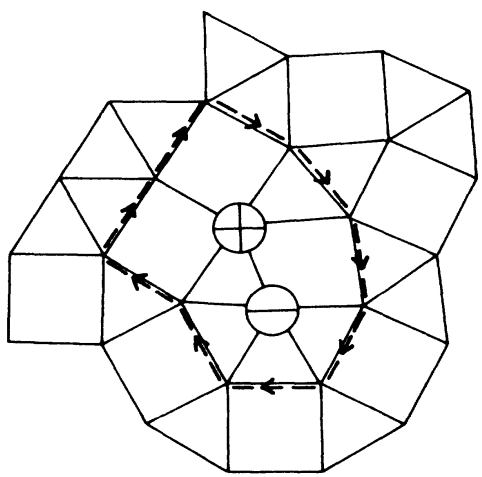

(c)

FIG. 5. (a) A neighboring pair of disclinations of strength $+1(\oplus)$ and $-1(\ominus)$, respectively. The kite-shaped quadrilateral in the middle is taken to be a square. (b) The same quadrilateral is taken to be two triangles divided by the dotted line. We have the same pair of disclinations, but at different positions. (c) Such a pair of disclinations forms a dislocation. As in ordinary two dimensional crystals, we can draw a Burgers circuit surrounding the dislocation (dashed lines). The sum of the lattice vectors along the circuit is the Burgers vector.
Figure 6 shows the network of Fig. 4(b) with the disclinations marked. There are three groups of dislocations labeled $A, B$, and $C$.

\section{Phason strain analysis}

A tiling free of disclinations and dislocations may still have random or systematic deviations from the ideal orientational symmetry. An elegant way to represent such fluctuations is to lift up the rigid structure into a higher-dimensional space in which it becomes a section of a periodic structure. ${ }^{38}$ In particular, a square and triangle tiling can be lifted into a four-dimensional hyperlattice which is a product of two triangular lattices, each being two dimensional, as mentioned in Sec. II A. To study the degree of order of a tiling, it is useful to resolve it another way into two mutually orthogonal subspaces: the "physical space" and "perpendicular space" whose vectors are denoted by $x^{\|}$and $x^{\perp}$, respectively. In the case of a square and triangle packing, the possible displacements of an atom to its nearest neighbors are the twelve unit vectors $\hat{\mathbf{e}}_{m}^{\|}$at angles $(2 \pi / 12)(m-1)$. Four of these vectors are independent over integers as shown in Fig. 7(a). To every coordinate of a vertex $x^{\|}$in the physical space

$$
\mathbf{x}^{\|}=\sum_{i=1}^{4} n_{i} \mathbf{e}_{i}^{\|}
$$

we can construct the corresponding vector $\mathbf{x}^{1}$ in the perpendicular space as

$$
\mathbf{x}^{\perp}=\sum_{i=1}^{4} n_{i} \mathbf{e}_{i}^{\perp},
$$

where $\mathbf{e}_{i}^{\perp}$ are defined in Fig. 7(b). In a perfect 12-fold quasiperiodic tiling produced by the cut-and-project method, $\mathbf{x}^{\perp}$ is virtually constant over the entire tiling, i.e., the hyperspace is flat in four dimensions. Since we are concerned with tilings which in some sense approximate the quasiperiodic tiling, we can write ${ }^{11}$ as an approximation,

$$
\mathbf{x}^{\perp} \simeq \mathbf{A} \mathbf{x}^{\|}+\text {const } .
$$

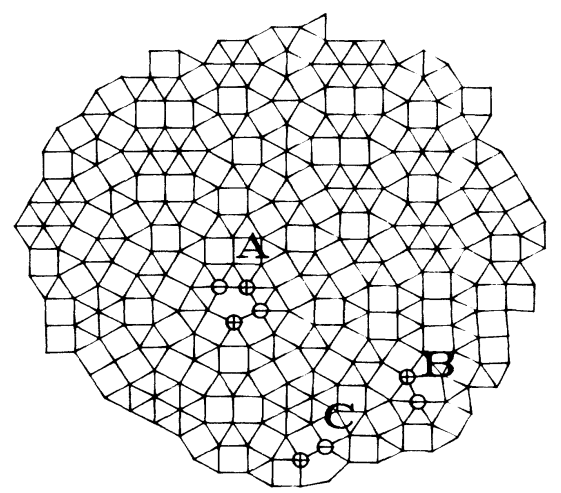

FIG. 6. A lattice network with disclinations marked. $\oplus$ and $\Theta$ represent a disclination of strength +1 and -1 , respectively. The three groups of dislocations are labeled as $A, B$, and $C$. 


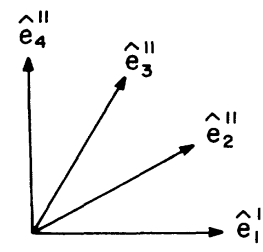

(a)

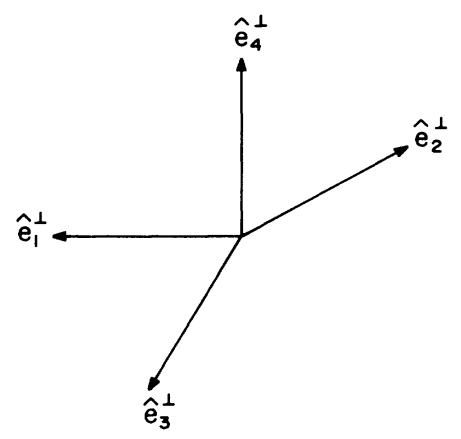

(b)

FIG. 7. (a) Basis vectors in physical space. (b) Basis vectors in perpendicular space.

A is called the phason strain,

$$
\mathbf{A}=\nabla_{\|} \mathbf{x}^{\perp} .
$$

If $\mathbf{A}$ is zero, the diffraction peaks coincide with that of a quasiperiodic tiling. Nonzero phason strain causes the diffraction peaks to shift. ${ }^{39}$ The sharpness and intensity of the diffraction maxima depend further on the fluctuation of $\mathbf{x}^{\perp}$, defined as

$$
\left.\delta \mathbf{x}^{12}=\langle| \mathbf{x}^{1}-\mathbf{A} \mathbf{x}^{\|}-\text {const }\left.\right|^{2}\right\rangle .
$$

Bragg peaks exist if $\delta x^{12}$ is finite as the volume goes to infinity. ${ }^{38}$ The effect of the fluctuations in $x^{\perp}$ is exactly analogous to that of atomic displacements $\mathbf{u}\left(\mathbf{x}^{\|}\right)$(phonons): (1) If $\delta x^{12}$ is infinite, then given the correlation function

$$
\left\langle\left|\mathbf{x}^{\perp}\left(\mathbf{x}^{\|}\right)-\mathbf{x}^{\perp}(\mathbf{0})\right|^{2}\right\rangle,
$$

one can find the shape of diffraction peaks; (2) if $\delta x^{12}$ is finite, then Bragg peaks exist, although with an intensity reduction analogous to the Debye-Waller factor.

For a rigid network of squares and triangles, we can represent the physical space vectors easily according to (9) by choosing an arbitrary point as the origin, and then (10) gives the perpendicular space vectors $\mathbf{x}_{i}^{\perp}$ for each (large) atom. The phason strain $\mathbf{A}$ can then be found by linear least-square fitting to (11). Applying such a procedure to the configuration in Fig. 1(b), we get

$$
\begin{aligned}
\delta \mathbf{x}^{12} & =1.101 \sigma_{L L}^{2}, \\
\mathbf{A} & =\left(\begin{array}{cc}
0.017 & 0.000 \\
0.000 & -0.003
\end{array}\right)
\end{aligned}
$$

The errors in the matrix elements are estimated to be about 0.001 .

However, this computation is complicated by the presence of frozen in defects when we apply it to the network in Fig. 6. We group neighboring disclinations into pairs, thereby defining dislocations (this is done by hand and there is some arbitrariness when there are four or more disclinations together). Let $\mathbf{B}_{j}$ be the Burgers vector of the $j$ th dislocation group, and define its position $\xi_{j}$ as the center of mass of the disclinations which constitute the dislocations. Each $\mathbf{B}_{j}$ is an integer combination of $\mathbf{e}_{i}^{\|}$and has a corresponding perpendicular space component $\mathbf{B}_{j}^{\perp}$ defined by $\mathbf{e}_{i}^{\|} \rightarrow \mathbf{e}_{i}^{\perp}$ in (8). To take into account the dislocations, the perpendicular space vector of each lattice point is corrected by

$$
\mathbf{x}^{\perp} \rightarrow \mathbf{x}^{\perp}-\sum_{j} \frac{\theta\left(\mathbf{x}^{\|}-\xi_{j}\right)}{2 \pi} \mathbf{B}_{j}^{\perp},
$$

where $\theta\left(\mathbf{x}^{\|}-\xi\right)$ is the angle subtended by $\mathbf{x}^{\|}$about the center of a dislocation at $\xi$. This procedure removes the dislocation poles from the hypersurface given by $\mathbf{x}^{\perp}\left(\mathbf{x}^{\|}\right)$, leaving only the phason fluctuations. For example, for the configuration shown in Fig. 6, the sum in (15) has three terms from the dislocation groups at positions labeled $A, B$, and $C$.

Using a linear least-squares fit, we find

$$
\begin{aligned}
& \delta \mathbf{x}^{12}=2.21 \sigma_{L L}^{2}, \\
& \mathbf{A}=\left(\begin{array}{cc}
-0.21 & -0.04 \\
0.03 & -0.12
\end{array}\right) .
\end{aligned}
$$

The errors in the matrix elements are estimated to be about 0.01. However, this is only the phason strain for one particular cooling process. To get a general idea of the magnitude and fluctuation of the phason strain for this kind of process, we have performed two more similar but independent runs. In order to present our results in a way independent of the particular coordinate system we chose, we decompose $\mathbf{A}$ in different representations of the rotation group, finding one two-dimensional representation and two copies of a one-dimensional representation. Thus we write $\mathbf{A}$ in the form

$$
\mathbf{A}=\alpha\left(\begin{array}{ll}
1 & 0 \\
0 & 1
\end{array}\right)+\beta\left(\begin{array}{cc}
0 & -1 \\
1 & 0
\end{array}\right)+\left(\begin{array}{cc}
\gamma & \mu \\
\mu & -\gamma
\end{array}\right) \text {. }
$$

Then, $|\alpha|,|\beta|$, and $\left(\gamma^{2}+\mu^{2}\right)^{1 / 2}$ are invariant under a rotation of $(2 \pi / 12) n$. Table I shows these values for the three different runs. It appears that $|\alpha|$ and $\left(\gamma^{2}+\mu^{2}\right)^{1 / 2}$ are of the order of 0.1 while $|\beta|$ is close to zero.

\section{Analysis of diffraction pattern}

In real quasicrystals, diffraction peaks are observed to be distorted and shifted. ${ }^{40,41}$ This has been interpreted as an effect of superimposing diffraction from regions with different phason strains. For a uniform (sometimes called "linear") phason strain field, that is one of form (11), the shift is given by ${ }^{42}$

$$
\Delta \mathbf{q}=\mathbf{A}^{T} \mathbf{q}^{\perp}
$$

where $\mathbf{A}^{T}$ denotes the transpose of $\mathbf{A}$. By measuring the

TABLE I. Invariant representation of phason strain matrices from three different cooling processes.

\begin{tabular}{ccc}
\hline \hline$|\alpha|$ & $|\beta|$ & $\left(\gamma^{2}+\mu^{2}\right)^{1 / 2}$ \\
\hline 0.165 & 0.035 & 0.045 \\
0.089 & 0.024 & 0.099 \\
0.043 & 0.005 & 0.095 \\
\hline \hline
\end{tabular}


peak shifts, we can find the phason strain matrix $\mathbf{A}$ by a linear least square fitting. Alternately, we can measure the position of a ring of 12 peaks $\mathbf{q}_{k}, k=1,2, \ldots, 12$ in the diffraction pattern. The phason strain matrix $\mathbf{A}$ is given by (see Appendix B)

$$
\mathbf{A}=\frac{1}{6\left|\mathbf{q}_{1}^{\perp}\right|^{2}}\left[\sum_{k=1}^{12} \mathbf{q}_{k} \mathbf{q}_{k}^{\perp}\right]^{T}
$$

Before we look at the diffraction pattern of the configuration in Fig. 4(b), we shall first analyze the perfect tiling of squares and triangles in Fig. 1(b). The lengths are scaled to the minimum of the Lennard-Jones potential (1) which is

$$
r_{\min }=2^{1 / 6} \sigma_{L L} \text {. }
$$

Since this lattice can be lifted up to four dimensions, each lattice point can be described in terms of four basis vectors as in (9). In reciprocal space, there are similarly four basis vectors $\mathbf{q}_{i}^{\|}$resulting from the product of the reciprocal lattices of two triangular lattices, whose magnitudes are

$$
\left|\mathbf{q}_{i}^{\|}\right|=\frac{2 \pi}{\sqrt{3} r_{\min }} .
$$

They are shown in Fig. 8(a). Hence, each peak can be labeled by four integer indices $\left(h_{1} h_{2} h_{3} h_{4}\right)$,

$$
\mathbf{Q} \|_{\left.h_{1} h_{2} h_{3} h_{4}\right)}=\sum_{i=1}^{4} h_{i} \mathbf{q}_{i}^{\|} .
$$

In analogy to the real space, there is a corresponding $\mathbf{Q}_{\left(h_{1} h_{2} h_{3} h_{3} h_{4}\right)}^{\perp}$ in perpendicular space given by

$$
\mathbf{Q}_{\left(h_{1} h_{2} h_{3} h_{4}\right)}^{\perp}=\sum_{i=1}^{4} h_{i} \mathbf{q}_{i}^{\perp},
$$

where $\mathrm{q}_{i}^{\perp}$ are shown in Fig. 8(b). Figure 9 shows the diffraction pattern of Fig. 1(b). It is very similar to the diffraction pattern of the dodecagonal phase of $\mathrm{V}-\mathrm{Ni}-\mathrm{Si}^{4}$.

Now, a square approximant structure as we use in Fig. 1 (a) is just the dodecagonal structure with a particular, rational phason strain. Therefore, the same formula (18) is valid for the peak shifts, which produce a diffraction pattern where all peaks belong to a square reciprocal lat-

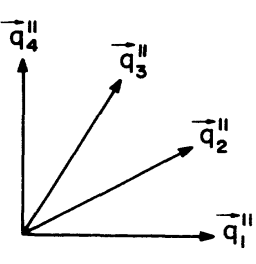

(a)

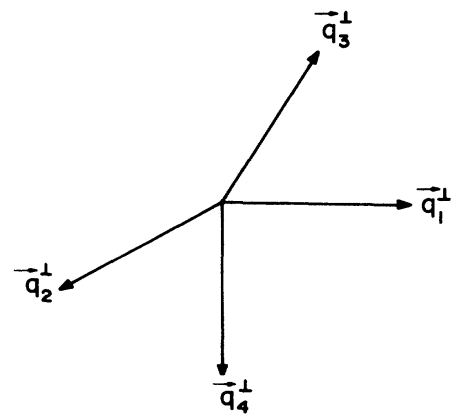

(b)
FIG. 8. Relation between $\mathbf{q}_{i}$ and $\mathbf{q}_{i}^{1}$. Note that this convention is consistent with Fig. 7. tice with basis vectors $\left(2 \pi / a_{\mathrm{sq}}\right) \hat{\mathbf{x}}$ and $\left(2 \pi / a_{\mathrm{sq}}\right) \hat{\mathbf{y}}$, where

$$
a_{\mathrm{sq}} \equiv(2+\sqrt{3})^{2} r_{\min }
$$

is the lattice constant of the square lattice. The phason strain corresponding to this can be found according to (19). Table II shows the indices, positions and the perpendicular space vector of the six peaks of the third visible ring in Fig. 9. The result is

$$
\mathbf{A}=\left(\begin{array}{cc}
0.017 & 0.000 \\
0.000 & -0.003
\end{array}\right) \text {. }
$$

This is only a weak phason strain. The peaks are shifted slightly but are not distorted. Note that this agrees with (14).

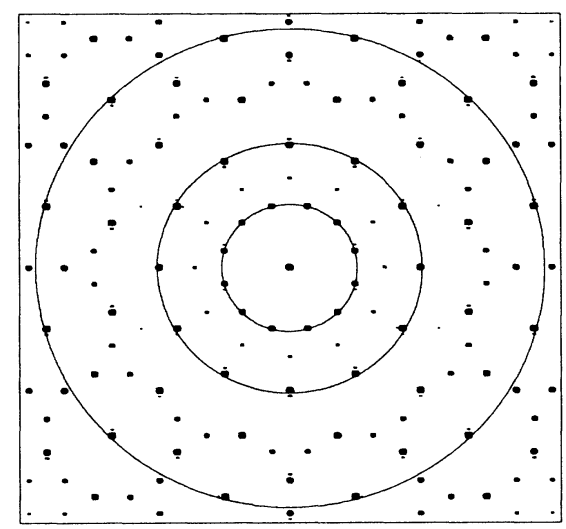

(a)

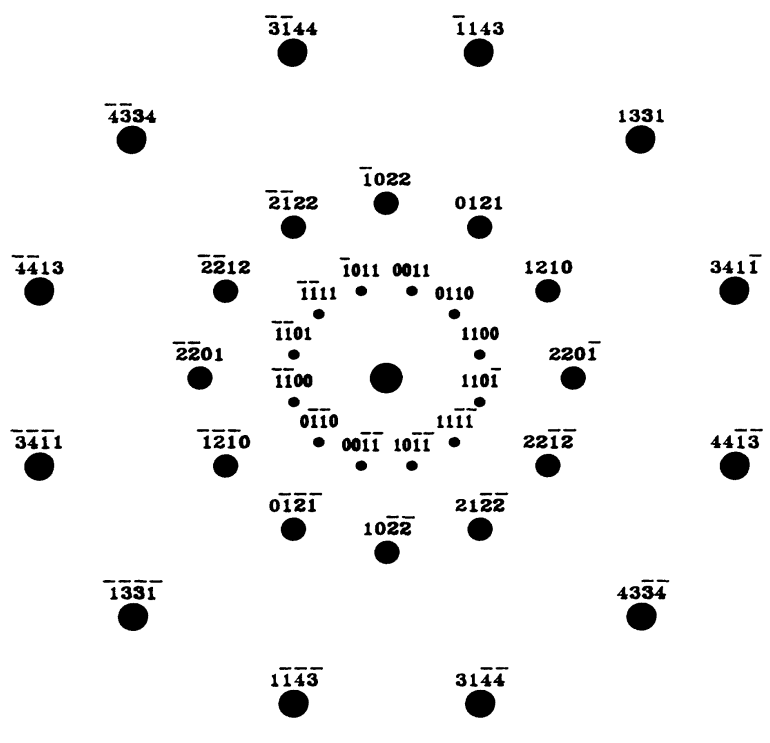

(b)

FIG. 9. (a) Diffraction pattern of the configuration in Fig. 1(b). The sides of the box are of length $50 / \sigma_{L L}$. It is plotted by putting a grid in the box and marking a dot at those grid points with $S(Q)$ above some threshold value. In this way the structures of the peaks are visible. (b) Indices of the three rings as marked in (a). 
TABLE II. Indices, position vectors $\mathbf{Q}$, perpendicular space vectors $\mathbf{Q}^{\perp}$ and the peak height $S(\mathbf{Q})$ for the peaks in Fig. 9(a).

\begin{tabular}{llll}
\hline \hline Index & \multicolumn{1}{c}{$\mathbf{Q} \sigma_{L L}$} & \multicolumn{1}{c}{$\mathbf{Q}^{\perp} \sigma_{L L}$} & $S(\mathbf{Q})$ \\
\hline $220 \overline{1}$ & $(12.076,0.000)$ & $(0.866,0.000)$ & 0.749 \\
1210 & $(10.433,6.032)$ & $(-0.750,-0.433)$ & 0.747 \\
0121 & $(6.038,10.443)$ & $(0.433,0.750)$ & 0.744 \\
$\overline{1} 022$ & $(0.000,12.065)$ & $(0.000,-0.866)$ & 0.743 \\
$\overline{2} \overline{1} 22$ & $(-6.038,10.443)$ & $(-0.433,0.750)$ & 0.744 \\
$\overline{2} \overline{2} 12$ & $(-10.433,6.031)$ & $(0.750,-0.433)$ & 0.747 \\
\hline \hline
\end{tabular}

Due to the nature of formation, one would expect that the phason strain field associated with the configuration in Fig. 4(b) to be much larger. Figure 10 shows its diffraction pattern. Table III shows the positions of the highest points of the peaks in the three rings marked in Fig. 10. By a linear least-square fitting to (18), the phason strain matrix is found to be

$$
\mathbf{A}=\left(\begin{array}{cc}
-0.23 & 0.07 \\
0.04 & -0.12
\end{array}\right)
$$

The errors in the matrix elements are estimated to be about 0.05. By comparing this with Sec. III D, we see that (25) and (16) are not quite the same, although not widely different either. Accurate determination of the positions of the peaks are undoubtedly affected by the peak structures. Isolated dislocations can distort the peaks whereas misoriented subgrains created by lines of dislocations can split the peaks. So the matrix A determined from the diffraction pattern must be less reliable in the presence of topological defects. A typical peak structure is shown in Fig. 11.

\section{CONCLUSION}

Using a simple, angular independent potential and standard Monte Carlo techniques, we can obtain a square and triangle tiling, although with frozen in defects which are unavoidable. Our model is more realistic than those

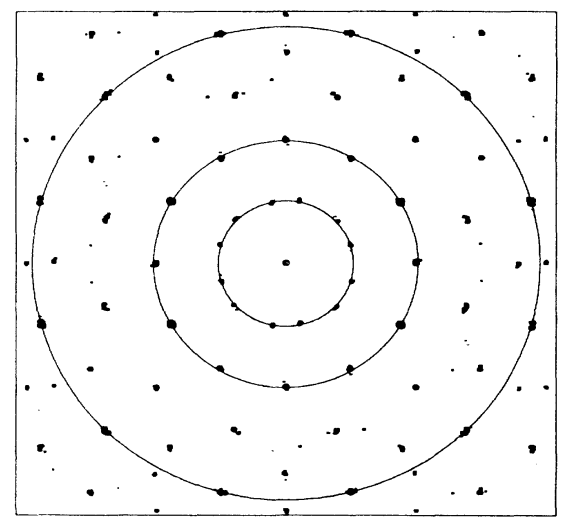

FIG. 10. Diffraction pattern of the configuration in Fig. 4(b). models based on rigid structures and the interaction potentials are more natural. We characterized the deviation of this tiling from perfect dodecagonal order by the phason strain matrix, which can be found by using either the real space or reciprocal space coordinates. Three independent freezing processes have been performed in order to get a general idea of the magnitude and fluctuations for the phason strain. We also monitored the system as it froze to observe the development of ordering. Although this atomic model has simple interaction potentials that are suitable for Monte Carlo simulations, as mentioned in Sec. II C the resulting equilibrium configurations are difficult to sample due to the highenergy barriers between different tilings. Hence, very long runs are necessary and this program is very demanding in computing resources. Furthermore, the use of free boundary conditions introduces surface effects (Sec. II C) which can be minimized only by using a larger system. Finite-size effect is also reflected in the fact that in Table I, the numbers are not strikingly similar in different runs. This is because to find a good value for $\mathbf{A}$, we need a

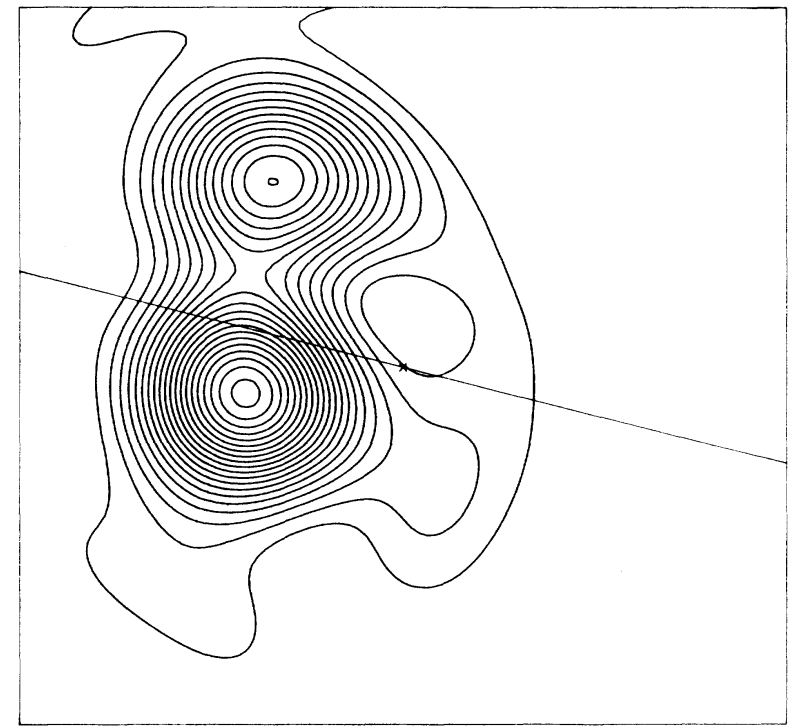

FIG. 11. Contour map of peak $(\overline{4} \overline{4} 13)$ in Fig. 10. The sides of the square box are $1.5 / \sigma_{L L}$. The main peak is split in two. The height of the peaks are 0.41 and 0.28 . 
TABLE III. Positions of the peaks in Fig. 10.

\begin{tabular}{ll}
\hline \hline Index & \multicolumn{1}{c}{$\mathbf{Q} \sigma_{L L}$} \\
\hline 1100 & $(6.010,1.753)$ \\
0110 & $(4.816,4.206)$ \\
0011 & $(1.258,6.175)$ \\
$\overline{1} 011$ & $(-1.300,6.001)$ \\
$\overline{1} \overline{1} 11$ & $(-4.729,4.353)$ \\
$\overline{1} 101$ & $(12.033,0.008)$ \\
$220 \overline{1}$ & $(10.727,5.932)$ \\
1210 & $(6.072,10.498)$ \\
0121 & $(-0.004,12.265)$ \\
$\overline{1} 022$ & $(-5.982,10.498)$ \\
$\overline{2} \overline{1} 22$ & $(-10.789,6.271)$ \\
$\overline{2} \frac{2}{2} 12$ & $(22.796,6.267)$ \\
$341 \overline{1}$ & $(16.791,16.445)$ \\
1331 & $(6.021,22.823)$ \\
$\overline{1} 143$ & $(-5.991,22.772)$ \\
$\overline{3} \frac{1}{1} 44$ & $(-16.774,16.524)$ \\
$\overline{4} \overline{3} 34$ & $(-22.814,5.977)$ \\
$\overline{4} \frac{1}{4} 13$ & \\
\hline
\end{tabular}

large system to average out the fluctuations in the perpendicular space. We will need to simulate a larger system before we can draw any definite conclusions.

It is interesting to compare our present model with the melting transition in ordinary two-dimensional crystals. ${ }^{43}$ In the latter case it is suspected, although without conclusive evidence, that it is a succession of two continuous phase transitions mediated by the unbinding of dislocations and disclinations, respectively. ${ }^{44-46}$ The intermediate phase between these two transitions is called the hexatic phase which possesses quasi long-range orientational order, but only short-range translational order. It has been speculated, in the context of Landau theories, that there might be such an orientationally ordered phase in quasicrystal phase diagrams. ${ }^{47}$ In our model, dislocations can have Burgers vectors smaller than an interatomic space, allowing reduced strain energy. This may make the unbinding of dislocation pairs easier and hence favor the Kosterlitz-Thouless type of melting transition. On the other hand, in our model the bond orientation is rotated only by $30^{\circ}$ around a single disclination, instead of $60^{\circ}$ as in ordinary two-dimensional crystals. This makes the dissociation of dislocations into free disclinations easier, thus favoring the first-order melting transition. We are not sure which of these competing effects dominates. So far, the most that can be said is that there is no particular evidence for a strong first-order transition. In any case, though, finite-size effects are likely to blur the distinction between first order and continuous transitions in a system of 400 particles such as ours, and so our results on the order of transition are necessarily inconclusive.

\section{ACKNOWLEDGMENTS}

We would like to thank Veit Elser, Joshua E. S. Socolar, Robert H. Swendsen, Y. Joanna Wong, and David C.
Wright for helpful discussions, and Laurence J. Shaw, Katherine J. Strandburg, and Michael Widom for comments on the manuscript. One of us (C.L.H.) was supported in part by National Science Foundation (NSF) Grant No. DMR-84-51921, by the IBM Corporation, and by Department of Energy (DOE) Grant No. DE-FG-287ER-45323. Two of us (P.W.L.) and (G.V.C.) were supported by NSF Grant No. DMR-85-16616. The computations were performed in Cornell National Supercomputer Facilities which are funded in part by the National Science Foundation, New York State, and IBM Corporation.

\section{APPENDIX A: FREQUENCY OF SQUARES AND TRIANGLES}

Consider a square-triangle tiling with dodecagonal symmetry. (The edge length will be taken to be unity here.) We claim (see Sec. II A) that the number of squares $N_{\mathrm{sq}}$ and the number of triangles $N_{\mathrm{tr}}$

$$
N_{\mathrm{sq}} / N_{\mathrm{tr}}=\sqrt{3} / 4 \text {. }
$$

Defining $n_{\mathrm{sq}}$ and $n_{\mathrm{tr}}$ as the number densities, per unit area, of squares and triangles, we see that (A1) implies

$$
\begin{aligned}
& n_{\mathrm{sq}}=\frac{1}{2}, \\
& n_{\mathrm{tr}}=2 / \sqrt{3} .
\end{aligned}
$$

(Thus, it is amusing to note, squares and triangles each occupy exactly $\frac{1}{2}$ of the total area.) The purpose of this appendix is to verify (A1) and (A2) in several ways.

\section{Ideal tiling made by inflation}

A tiling of squares and triangles can be produced by various modifications of the method of inflation. ${ }^{28} \mathrm{We}$ start with a square-triangle tiling, and decorate it by squares and triangles which are smaller by a factor $(2+\sqrt{3})$ to make a new tiling of the smaller squares and triangles; we then rescale these to the original size, and continue the process iteratively. Figure 1(a) is made by two iterations of this process where the starting configuration was one square per cell with the vertices at the corners. More specifically, each vertex is replaced by a dodecagon consisting of 6 squares and 12 triangles, as in Fig. 2, in such a way that the dodecagons from neighboring vertices of the original lattice just touch along one edge. For each dodecagon, we may choose between two possible orientations. We can choose them deterministically according to the orientation of the nearest-neighbor shell of the starting tiling, generating a quasiperiodic structure. Alternatively, we can choose them at random, producing a restricted ensemble of random tilings with an entropy per vertex of

$$
\ln 2 /(6+4 \sqrt{3}) \cong 0.0536 \text {. }
$$

The leftover space is filled by one small triangle in the triangle, and by one small square plus four small triangles in the square. Thus, (square) $\rightarrow 7$ (square) +16 (triangle) and (triangle) $\rightarrow 7$ (triangle) +3 (square), i.e., 


$$
\left(\begin{array}{c}
N_{\mathrm{sq}}^{\prime} \\
N_{\mathrm{tr}}^{\prime}
\end{array}\right)=\left(\begin{array}{cc}
7 & 3 \\
16 & 7
\end{array}\right)\left[\begin{array}{l}
N_{\mathrm{sq}} \\
N_{\mathrm{tr}}
\end{array}\right) .
$$

The dominant eigenvector is $(\sqrt{3}, 4)$ with eigenvalue $(2+\sqrt{3})^{2}$. Clearly, (A 1$)$ holds in the limit of many iterations.

\section{From rhombic tiling with dodecagonal symmetry}

A more general approach is to consider the tiling as the projection of a faceted two-dimensional surface made up of faces of a regular lattice in a $D$-dimensional hyperspace. ${ }^{9}$ The formulas derived in Ref. 9, however, apply only to tilings of rhombi, projected from hypercubic lattices. For dodecagonal symmetry, such a lattice has been described ${ }^{48}$ with $D=6$.

The hyperlattice is constructed as follows: we let

$$
\mathbf{e}_{\alpha} \equiv \mathbf{e}_{\alpha}^{\|}+\mathbf{e}_{\alpha}^{1}
$$

be the basis vectors in the hyperspace. The normalization here is

$$
\left|\mathbf{e}_{\alpha}\right|=(D / d)^{1 / 2} \text {, }
$$

where $d=2$ is the ordinary spatial dimension.

Given a phason strain matrix $\mathbf{A}$, we define vectors

$$
\mathbf{m}_{\alpha}=\mathbf{e}_{\alpha}^{\|}+\mathbf{A}^{T} \mathbf{e}_{\alpha}^{\perp} .
$$

(This differs from Ref. 9, Eq. (2.5), only because our normalization of $\left\{\mathbf{e}_{\alpha}^{\perp}\right\}$ differs). We can think of (A5) as a parametrization of the 2-plane embedded in hyperspace. It then follows from $\mathbf{x}^{\perp}=\mathbf{A} \mathbf{x}^{\|}$and $\mathbf{x} \equiv \mathbf{x}^{\perp}+\mathbf{x}^{\|}$that

$$
\mathbf{e}_{\alpha} \cdot \mathbf{x}=\mathbf{m}_{\alpha} \cdot \mathbf{x}^{\|} .
$$

Let us choose a particular type and orientation of tile: all such tiles are projected from 2 -faces of the same orientation in the hyperspace lattice; counting the chosen tiles is the same as counting the chosen 2 -faces in the faceted surface.

We want to count the chosen tiles in a domain in physical space of area $S^{\|}$. This domain projects up to a patch of the faceted hypersurface which (if phason strain is well defined) is, on average, planar. Let us reproject this patch onto one of the coordinate 2-planes of the hyperspace lattice, which is a regular lattice of 2-cells; let $S_{\alpha \beta}^{*}$ be the area of the reprojected patch on the $(\alpha, \beta)$ coordinate plane. It can be shown from (A6) that the ratio of reprojected area $S_{\alpha \beta}^{*}$ on the $(\alpha, \beta)$ coordinate plane, to area $S^{\|}$in physical space, is

$$
S_{\alpha \beta}^{*} / S^{\|}=\left|\mathbf{m}_{\alpha} \times \mathbf{m}_{\beta}\right| /\left|\mathbf{e}_{\alpha} \times \mathbf{e}_{\beta}\right| .
$$

(By $\left|\mathbf{e}_{\alpha} \times \mathbf{e}_{\beta}\right|$, we mean more precisely $\left[\left|\mathbf{e}_{\alpha}\right|^{2}\left|\mathbf{e}_{\beta}\right|^{2}\right.$ $\left.-\left(\mathbf{e}_{\alpha} \cdot \mathbf{e}_{\beta}\right)^{2}\right]^{1 / 2}$.)

Then each face oriented parallel to the chosen coordinate 2-plane must reproject to a face of that 2-plane. Also, the coordinate planes are mutually orthogonal, so all other kinds of face reproject to nothing. The number of faces of the chosen orientation in the patch is the same as the number of 2-cells in the reprojected patch, hence equal to its area $S_{\alpha \beta}^{*}$ divided by the area $S_{\mathrm{sq}}^{*}=3$ of one square cell.
Now we know the density of the corresponding tiles in the tiling: putting together the above results, we get

$$
n_{\alpha \beta}=\left|\mathbf{m}_{\alpha} \times \mathbf{m}_{\beta}\right| /\left(\left|\mathbf{e}_{\alpha} \times \mathbf{e}_{\beta}\right| S_{\alpha \beta}^{*}\right),
$$

where the denominator is 9 for any $\alpha \neq \beta$ for our 6D hypercubic lattice of lattice constant $\sqrt{3}$. (A8) is the same as Eq. (2.6) of Ref. 9.

It can be shown (see Ref. 9, and also part 3 of this appendix) that nonzero phason strain implies that a given type of rhombus appears more often in some orientations than in others. Therefore, our assumption of 12-fold symmetry implies $\mathbf{A}=0$; then (A8) states that the frequency of a rhombus type is just proportional to the area of one of the rhombi of that type.

The 12-fold symmetric rhombus tiling has three types of rhombus:

(i) $30^{\circ}$ rhombi, e.g.,

$$
n_{12}=\left|\mathbf{m}_{1} \times \mathbf{m}_{2}\right| / 9=\frac{1}{18} \text {. }
$$

There are six possible orientations, so the total frequency is

$$
n_{\pi / 6}=6\left(\frac{1}{18}\right)=\frac{1}{3} \text {. }
$$

(ii) $60^{\circ}$ rhombi, e.g.,

$$
n_{13}=\left|\mathbf{m}_{1} \times \mathbf{m}_{3}\right| / 9=1 /(6 \sqrt{3}) \text {. }
$$

There are again six possible orientations, so the total frequency is

$$
n_{\pi / 3}=6 /(6 \sqrt{3})=1 / \sqrt{3} \text {. }
$$

(iii) $90^{\circ}$ rhombi, i.e., squares, e.g.,

$$
n_{14}=\left|\mathbf{m}_{1} \times \mathbf{m}_{4}\right| / 9=\frac{1}{9} \text {. }
$$

There are three possible orientations, so the total frequency is

$$
n_{\pi / 2}=3\left(\frac{1}{9}\right)=\frac{1}{3} \text {. }
$$

To make this rhombus tiling into a tiling of squares and triangles, (i) we divide each $60^{\circ}$ rhombus into two triangles (ii) where one vertex is shared by the obtuse corners of two $30^{\circ}$ rhombi and the acute angle of a $60^{\circ}$ rhombus so as to form a flattened hexagon, we can combine and redivide the rhombi to make a square with triangles on two opposite faces. Since a $60^{\circ}$ rhombus is equivalent to two triangles, we can regard the second process as grouping a pair of $30^{\circ}$ rhombi to make a square. (However, in the directly projected tiling, not all $30^{\circ}$ rhombi are in pairs; it is necessary to move them through the tiling, by reshuffling groups such as the irregular hexagon around a vertex shared by the obtuse corners of a $30^{\circ}$ rhombus, a $60^{\circ}$ rhombus and a square.) This gives

$$
n_{\mathrm{tr}}=2 n_{\pi / 3}=2 / \sqrt{3}
$$

and

$$
n_{\mathrm{sq}}=\frac{1}{2} n_{\pi / 6}+n_{\pi / 2}=\frac{1}{2},
$$

in agreement with (A2). 


\section{General argument}

The above are special cases. Here, we derive the general formula for the density of each orientation of square and triangle, valid for arbitrary values of the phason strain, and with no constraint on how the tiling was constructed. This result for squares and triangles is analogous to (A8) for rhombi, and its derivation is based on the same idea as that of (A8) in Ref. 9. There is a minor technical complication which necessitates redoing the derivation in Ref. 9: a general square-triangle tiling requires a noncube hyperlattice.

The tiling is now described as a projection from a four dimensional hyperlattice (see Sec. II A and Refs. 26 and 27). Hyperspace basis vectors are again defined by (A3), but now $\mathrm{e}^{\perp}$ belongs to a two-dimensional space. If $\mathrm{e}_{\alpha}^{\perp}$ are normalized to be unit vectors, then (A4) again holds so $\left|\mathbf{e}_{\alpha}\right|=\sqrt{2}$ for this lattice.

It is important to note that the basis vectors in hyperspace are not orthogonal: in fact, $\mathbf{e}_{1}$ and $\mathbf{e}_{3}$ make a triangular lattice (of lattice constant $\sqrt{2}$ ); similarly, $\mathbf{e}_{2}$ and $\mathbf{e}_{4}$ form a triangular lattice, orthogonal to the first one. The four-dimensional lattice is the product of these two triangular lattices, in the same way as the stacked triangular lattice in three dimensions is the product of a triangular lattice and a one-dimensional lattice. The triangles in real space are projected from triangular faces in one of these triangular planes, while the squares in real space are projected from square faces in a plane spanned by one basis vector from each of the triangular lattices.

For triangles, the arguments in part 2 carries through. Only one kind of triangle projects onto a triangular coordinate plane, so the number of faces is determined by dividing out the area of triangle $S_{\mathrm{tr}}^{*}=\sqrt{3} / 2$ on the coordinate plane.

Let $n_{\mathrm{tr}}^{13}$ be the density of $(1,3)$ triangles (those spanned by vectors $\mathbf{e}_{1}$ and $\mathbf{e}_{3}^{\|}$). There are two such orientations, related by an inversion [similarly there are two kinds of $(2,4)$ triangles]. The total number in our patch is $N_{\mathrm{tr}}^{13}=A_{13}^{*} / A_{\mathrm{tr}}^{*}$, therefore,

$$
n_{\mathrm{tr}}^{13}=A_{13}^{*} /\left(A^{\|} A_{\mathrm{tr}}^{*}\right)=\frac{2}{3}\left|\mathbf{m}_{1} \times \mathbf{m}_{3}\right|,
$$

and similarly for the two other kinds of triangles. ${ }^{49}$

The frequency of the squares is a more complicated case. Each $(1,4)$ square projects to a square of area $\boldsymbol{A}_{\mathrm{sq}}^{*}=2$ in the $(1,4)$ plane. However, the two other square faces have nonzero projections onto this plane, since

$$
\cos \theta_{13}=\cos \theta_{24}=\frac{1}{2},
$$

where $\theta_{\alpha \beta}$ is the angle between the basis vectors $\mathbf{e}_{\alpha}$ and $\mathbf{e}_{\beta}$. Thus, the $(2,5)$ and $(0,3)$ squares $^{50}$ also project on the
$(1,4)$ plane to squares of side $1 / 2 \mathrm{e}_{1}$ and $1 / 2 \mathrm{e}_{2}$, with area $1 / 4 A_{\mathrm{sq}}^{*}$. Consequently, all we can derive is

$$
n_{14}+\frac{1}{4}\left(n_{03}+n_{25}\right)=\left|\mathbf{m}_{1} \times \mathbf{m}_{4}\right| / 4 .
$$

Together with the corresponding linear equations from the two other kinds of square coordinate planes, of course, we can eliminate to finally get

$n_{14}=\frac{5}{18}\left|\mathbf{m}_{1} \times \mathbf{m}_{4}\right|-\frac{1}{18}\left|\mathbf{m}_{0} \times \mathbf{m}_{3}\right|-\frac{1}{18}\left|\mathbf{m}_{2} \times \mathbf{m}_{5}\right|$.

For the case of 12-fold symmetry, $\mathbf{A}=\mathbf{0}$ as noted above so that (A9) gives $n_{\mathrm{tr}}^{24}=n_{\mathrm{tr}}^{13}=1 / \sqrt{3}$, and (A10) gives

$$
n_{\mathrm{sq}}^{14}=n_{\mathrm{sq}}^{25}=n_{\mathrm{sq}}^{03}=\frac{1}{6}
$$

making total densities in agreement with (A2).

\section{APPENDIX B: PHASON STRAIN MATRIX FROM POSITIONS OF DIFFRACTION PEAKS}

Consider a ring of twelve peaks in the diffraction pattern

$$
\mathbf{q}_{k}=\mathbf{q}_{k}+\mathbf{A}^{T} \mathbf{q}_{k}^{\perp} \quad k=1,2, \ldots, 12,
$$

where $\mathbf{q}_{k}$ are the unshifted position of the peaks, $\mathbf{q}_{k}$ are their measured positions. We can form the dyad

$$
\mathbf{S} \equiv \sum_{k=1}^{12} \mathbf{q}_{k} \mathbf{q}_{k}^{\perp}=\sum_{k=1}^{12} \mathbf{q}_{k} \mathbf{q}_{k}^{\perp}+\mathbf{A}^{T} \sum_{k=1}^{12} \mathbf{q}_{k}^{\perp} \mathbf{q}_{k}^{\perp} .
$$

One can show that

$$
\sum_{k=1}^{12} \mathbf{q}_{k} \mathbf{q}_{k}^{1}=0
$$

Let

$$
\mathbf{S}^{\perp} \equiv \sum_{k=1}^{12} \mathbf{q}_{k}^{\perp} \mathbf{q}_{k}^{\perp}
$$

Since $\mathbf{S}^{\perp}$ cannot be associated with any particular direction in the perpendicular space

$$
\mathbf{S}^{\perp}=L \mathbf{I},
$$

where $\mathbf{I}$ is the identity matrix, and

$$
\begin{aligned}
L & =\frac{1}{2} \operatorname{Tr}\left(\mathbf{S}^{\perp}\right) \\
& =6\left|\mathbf{q}_{1}^{\perp}\right|^{2} .
\end{aligned}
$$

Hence, it follows that

$$
\mathbf{A}=\frac{1}{6\left|\mathbf{q}_{1}^{\perp}\right|^{2}} \mathbf{S}^{T} \text {. }
$$

${ }^{*}$ Present address.

${ }^{1}$ N. Wang, H. Chen, and K. H. Kuo, Phys. Rev. Lett. 59, 1010 (1987).

${ }^{2}$ L. Bendersky, Phys. Rev. Lett. 55, 1461 (1985).

${ }^{3}$ T. Ishimasa, H-U Nissen, and Y. Fukano, Phys. Rev. Lett. 55,
511 (1985).

${ }^{4} \mathrm{H}$. Chen, K. H. Kuo, and D. X. Li, in the Fourth China-Japan Seminar on Electron Microscopy, Kunming 1987 (unpublished).

${ }^{5}$ D. Shechtman, I. Blech, D. Gratias, and J. W. Cahn, Phys. 
Rev. Lett. 53, 1951 (1984).

${ }^{6}$ P. Sainfort, B. Dubost, and A. Dubus, C.R. Acad. Sci. Paris, 301, 689 (1985).

${ }^{7}$ D. Levine and P. J. Steinhardt, Phys. Rev. Lett. 53, 2477 (1984).

${ }^{8}$ V. Elser, Phys. Rev. Lett. 54, 1730 (1985).

${ }^{9}$ C. L. Henley, J. Phys. A 21, 1649 (1988).

${ }^{10}$ P. Sainfort and B. Dubost, J. Phys. (Paris) Colloq. 47, C3-321 (1986).

${ }^{11} \mathrm{~V}$. Elser, Proceedings of the XVth International Colloquium on Group Theory in Physics, edited by R. Gilmore and D. H. Feng (World Scientific, Singapore, 1987).

${ }^{12}$ C. L. Henley, Comments Cond. Mat. Phys. 13, 59 (1987).

${ }^{13}$ P. W. Stephens and A. J. Goldman, Phys. Rev. Lett. 56, 1168 (1986).

${ }^{14}$ P. A. Heiney, P. A. Bancel, P. M. Horn, J. L. Jordan, S. Laplaca, J. Angilello, and F. W. Gayle, Science 238, 660 (1987).

${ }^{15} \mathrm{P}$. W. Stephens and A. J. Goldman (unpublished). The pictures of their model are published in Ref. 14.

${ }^{16}$ V. Elser and C. L. Henley, Phys. Rev. Lett. 55, 2883 (1985).

${ }^{17}$ P. Guyot and M. Audier, Philos. Mag. B 52, L15 (1985).

${ }^{18}$ C. L. Henley and V. Elser, Philos. Mag. B 53, L59 (1986).

${ }^{19}$ Y. J. Wong and G. V. Chester, Phys. Rev. B 35, 3506 (1987).

${ }^{20} \mathrm{~F}$. Lançon, L. Billard, and P. Chaudhari, Europhys. Lett. 2, 625 (1986). Also F. Lançon and L. Billard, J. Phys. (Paris) 49, 249 (1988).

${ }^{21}$ M. Widom, K. J. Strandburg, and R. H. Swendsen, Phys. Rev. Lett. 58, 706 (1987).

${ }^{22}$ F. C. Frank and J. S. Kasper, Acta Crystallogr. 12, 483 (1959).

${ }^{23}$ D. X. Li and K. H. Kuo, Acta Crystallogr., Sect. B 42, 152 (1986).

${ }^{24}$ Q. B. Yang and W. D. Wei, Phys. Rev. Lett. 5, 1020 (1987).

${ }^{25}$ H. Chen, D. X. Li, and K. H. Kuo, Phys. Rev. Lett. 60, 1645 (1988). It is interesting to note that the high resolution images of their Fig. 2 shows almost no adjacent squares, as in our model if we take a larger $\epsilon_{S S}$ (see Sec. II C).

${ }^{26}$ N. Niizeki and H. Mitani, J. Phys. A 20, L405 (1987).

${ }^{27}$ V. E. Korepin, F. Gähler, and J. Rhyner (unpublished).

${ }^{28}$ P. Stampfli, Helv. Phys. Acta 59, 1260 (1986).

${ }^{29}$ H. Kawamura, Prog. Theor. Phys. 70, 352 (1983).
${ }^{30} \mathrm{M}$. Widom (unpublished).

${ }^{31}$ In fact, if we forbid neighboring squares in a square and triangle tiling, then connecting the centers of the squares gives a new tiling of squares, triangles, and hexagons with edges enlarged by a factor $1 / 2(\sqrt{3}+1)$, and with some matching rules. This obviously has less entropy than a square and triangle tiling of the same large edges. So the constraint costs more than a factor $(\sqrt{3}+1 / 2)^{2}$ in entropy.

${ }^{32}$ C. L. Henley, Phys. Rev. B 34, 797 (1986).

${ }^{33}$ C. L. Henley (unpublished).

${ }^{34}$ F. W. Dewette, R. E. Allen, D. S. Hughes, and A. Rahman, Phys. Lett. 29, A548 (1969).

${ }^{35}$ J. E. S. Socolar, T. C. Lubensky, and P. J. Steindhardt, Phys. Rev. B 3, 3345 (1986).

36J. Bohsung and H.-R. Trebin, Phys. Rev. Lett. 58, 1204 (1987).

${ }^{37}$ D. Levine, T. C. Lubensky, S. Ostlund, S. Ramaswamy, P. J. Steinhardt, and J. Toner, Phys. Rev. Lett. 54, 1520 (1985).

${ }^{38}$ V. Elser, Phys. Rev. B 32, 4892 (1985).

${ }^{39}$ J. E. S. Socolar and D. C. Wright, Phys. Rev. Lett. 59, 221 (1987).

${ }^{40}$ M. Tanaka, M. Terauchi, K. Hirage, and M. Hirabayashi, Ultramicroscopy 17, 279 (1985).

${ }^{41}$ P. A. Bancel and P. A. Heiney, J. Phys. (Paris) Colloq., 47, C3-341 (1986).

${ }^{42}$ T. C. Lubensky, J. E. S. Socolar, P. J. Steinhardt, P. A. Bancel, and P. A. Heiney, Phys. Rev. Lett. 57, 1440 (1986).

${ }^{43}$ K. H. Strandburg, Rev. Mod. Phys. 60, 161 (1988).

${ }^{44}$ J. M. Kosterlitz and D. J. Thouless, J. Phys. C 6, 1181 (1973).

${ }^{45}$ B. J. Halperin and D. R. Nelson, Phys. Rev. Lett. 41, 121 (1978).

${ }^{46}$ A. P. Young, Phys. Rev. B 19, 1855 (1979).

${ }^{47}$ M. V. Jarić, Phys. Rev. Lett. 55, 607 (1985).

${ }^{48}$ For example, F. Gähler and J. Rhyner, J. Phys. A 19, 267 (1986).

${ }^{49}$ Note that the $(3,5)$ triangles are the same as the $(1,3)$ triangles, but since $\mathbf{e}_{1}+\mathbf{e}_{5}=\mathbf{e}_{3}$, and similarly $\mathbf{m}_{1}+\mathbf{m}_{5}=\mathbf{m}_{3}$, the formula indeed gives the same answer $n_{\mathrm{tr}}^{13}=n_{\mathrm{tr}}^{15}=n_{\mathrm{tr}}^{35}$ independent of which basis vectors we chose to use.

${ }^{50}$ Note that $\mathbf{e}_{0} \equiv \mathbf{e}_{12}$. 